\title{
Beyond the Metrological Viewpoint
}

\author{
Jean Baccelli* \\ Munich Center for Mathematical Philosophy
}

December 7, 2018

\begin{abstract}
The representational theory of measurement (RTM) has long been the central paradigm in the philosophy of measurement. Such is not the case anymore, partly under the influence of the critique according to which RTM offers too poor descriptions of the measurement procedures actually followed in science. This can be called the metrological critique of RTM. I claim that the critique is partly irrelevant. This is because, in general, RTM is not in the business of describing measurement procedures, be it in idealized form. To support this claim, I present various cases where RTM can be said to investigate measurement without providing any measurement procedure. Such limit cases lead to a better understanding of the RTM project. They also illustrate some of the questions which the philosophy of measurement can explore, when it is ready to go beyond the metrological viewpoint.
\end{abstract}

${ }^{*}$ For helpful comments, I am grateful to two reviewers, Neil Dewar, Sam Fletcher, Stephan Hartmann, Conrad Heilmann, Alistair Isaac, Luca Mari, Philippe Mongin, Louis Narens, Gualtiero Piccinini, Elina Vessonen, and Jo Wolff. I also thank audiences at BSPS'17 (Edinburgh), EPSA'17 (Exeter), "Measurement at the Crossroads" (Paris, 2018), and PSA'18 (Seattle). All errors and omissions are mine. 


\section{Introduction}

The representational theory of measurement (henceforth, RTM-especially Krantz et al., 1971; Suppes et al., 1989; Luce et al., 1990) has long been the central paradigm in the philosophy of measurement. RTM is mainly concerned with providing the various possible forms of measurement with mathematical foundations. It tackles this task by employing what has become its signature method of enquiry, namely, representation theorems. On the received view, RTM thus examines idealized measurement procedures. It simplifies measuring to assigning numbers, or constructing scales (e.g., Krantz et al., 1971, p. 9, 102). It investigates when this can be well-defined.

RTM has largely lost its influence in the philosophy of measurement. Dissatisfaction has grown with its idealization of measurement procedures. One can find such dissatisfaction expressed throughout the history of RTM, sometimes even by direct contributors to this literature (e.g., Stevens, 1968, p. 854; Niederée, 1992, p. 245). But the most forceful expression has been recently inspired by the metrology community. Metrology is officially defined as "the science of measurement and its application[s]" (Joint Committee for Guides in Metrology, 2008, p. 16). It is primarily a field concerned with the design, standardization, and use of measuring instruments. It thus embodies an extremely careful attention to the actual implementation of measurement procedures. It has also recently inspired influential contributions to the philosophy of science (as evidenced in, e.g., Mitchell et al., 2017). Metrology-inspired philosophers of measurement deplore the fact that RTM 'took 'measurement' to be synonymous with either 'number assignment' (...) or 'scale construction', and neglected the 'applied' aspects of measurement such as accuracy, precision, error, uncertainty, and calibration (...)" (Tal, 2013, p. 1164). They recommend paying much closer attention to the particulars of the measurement procedures followed in actual scientific practice. They advocate going "beyond the representational viewpoint" (Mari, 2000) to get richer insights on measurement.

Accordingly, call the following "the metrological critique": RTM poorly describes the measurement procedures actually followed in science, and thereby it offers the philosophy of science limited insights on measurement. There is much to grant in this critique. RTM alone is not sufficient to provide a complete account of measurement in the sciences. Other takes on measurement, such as those inspired by metrology, are necessary to complement what RTM can deliver. However, one important preliminary question is whether RTM really is in the business of describing measurement procedures, be it in idealized form. I take the metrological critique to be premised on a positive answer to this question. More generally, the vivid contrast between metrology and RTM constitutes a welcome occasion to revisit this received view. What is at stake is not only the correct interpretation of the RTM project - a matter of long-standing debates. It is also the possible directions 
for the philosophy of measurement, at a time when the metrological viewpoint dominates. When philosophers of science reflect upon measurement, should they be concerned with, more specifically, measurement procedures?

My main claim will be that, in general, RTM is not in the business of describing measurement procedures, be it in idealized form. To support this claim, I will present various cases where RTM can be said to investigate measurement without providing any measurement procedure. Such limit cases reveal that the RTM project is even more profoundly different from the metrological project than currently understood in the literature. On the alternative view which I will sketch, RTM is concerned with measurability, rather than measuring, and some of its results are remarkable precisely in that they illustrate that the former should not be reduced to the latter. This is naturally not an attack on the project of a metrology-inspired philosophy of science, let alone the research agenda of the metrology community. This is a defense of RTM against the metrological critique, to the effect that the critique is at least partly irrelevant. Besides, the polemical twist is essentially for vividness. What matters most is that taking the metrological critique into account leads to refining the received interpretation of the RTM project and to highlighting the existence of fundamentally non-metrological questions in the philosophy of measurement. Consider this an illustrated invitation to go, whenever relevant, "beyond the metrological viewpoint".

Several other authors have already, in the context of general discussions of the interpretations of RTM, offered indirect defenses of RTM against the metrological critique. See especially Narens and Luce, 1993; Decoene et al., 1995; Narens, 2002 (Sec. 5.3); Heilmann, 2015; and Vessonen, forthcoming. However, none of these discussions is as direct and as focused as mine, and none has followed the argumentative strategy sketched above. Unlike the above scholars, I emphasize the developments of RTM that do not square with the apparently conciliatory view according to which RTM is committed to investigating idealized measurement procedures, in contrast with the actual measurement procedures to be found in scientific practice. A related difference, the relevance of which will appear along the way, is that I emphasize developments of RTM that are not to be found in the pioneering volume of Krantz et al., 1971. By contrast, the above scholars do not sufficiently distance themselves from the widespread but outdated view according to which, as far as the philosophy of measurement is concerned, this particular contribution summarizes RTM without loss of generality.

The core of the paper is comprised of four short sections. Each section is organized around one illustrative theorem from the RTM literature. None of the theorems can be here discussed in great detail; but for my purposes, it is enough to give a sense of what kind of results they are. As a necessary preliminary, Section 1 recalls the fundamentals of the RTM approach. The subsequent sections discuss various ways in which the RTM approach can be said to investigate measurement without providing any measurement proce- 
dure. I will successively examine three cases. For brevity, I will call them the cases of "unknown" (Sec. 2), "irrelevant" (Sec. 3), and "unspecified" (Sec. 4) measurement procedures. A brief conclusion ensues.

\section{The Representational Approach in a Nutshell}

It is best to recall the fundamentals of the RTM approach by means of an example. The example introduced and elaborated upon next has been of great importance throughout the history of RTM. ${ }^{1}$ It permits illustrating not only the spirit of the RTM approach, but also, as a natural starting point, the received view according to which RTM proceeds by investigating idealized measurement procedures. The result is known as the Hölder theorem (for a full statement and a proof of the below variant of the theorem, which is the most accessible of all, see, e.g., Krantz et al., 1971, Thm. 3.1, p. 74).

Let $X$ be a non-empty set, $\succcurlyeq$, a binary relation over $X, \oplus$, a binary operation (i.e., a ternary relation) over $X$. For concreteness, one can interpret $X$ as a set of rigid rods, $\succcurlyeq$, as the ordering of these rods in terms of their respective lengths, $\oplus$, as the operation consisting in the concatenation of two rods. Another intuitive interpretation is with reference to the measurement of weight thanks to an equal-arm pan balance. Denote by $\mathcal{X}=\langle X, \succcurlyeq, \oplus\rangle$ the relational structure thus constituted, i.e., the set $X$ equipped with the various relations defined over it. The Hölder theorem states that $\mathcal{X}$ satisfies a distinguished set of properties - namely, it qualifies as a so-called "closed positive concatenation" structure -if and only if there exists a function $\phi: X \rightarrow \mathbb{R}_{>0}$ such that, for any $w, x, y, z \in X$, the following representing conditions hold:

$$
\begin{aligned}
& \text { i) } x \succcurlyeq y \Leftrightarrow \phi(x) \geq \phi(y) \text {; } \\
& \text { ii) } w \oplus x \succcurlyeq y \oplus z \Leftrightarrow \phi(w)+\phi(x) \geq \phi(y)+\phi(z) .
\end{aligned}
$$

This means that the length ordering of the rigid rods in $X, \succcurlyeq$, is represented by the usual ordering $\geq$ over the real numbers delivered by $\phi$ and furthermore that the concatenation operation $\oplus$ is represented by the usual addition operation + . The theorem is completed by the uniqueness proposition according to which $\psi: X \rightarrow \mathbb{R}_{>0}$ also satisfies i) and ii) if and only if $\psi=\alpha \phi$, for some $\alpha \in \mathbb{R}_{>0}$. The Hölder theorem thus offers mathematical foundations for some of the customary ways in which scientists measure length, weight, and still other comparable attributes.

Generally speaking, a representation result like the Hölder theorem is composed of the following elements, which can be considered the hallmarks of the RTM approach. On the one hand, take a qualitative relational structure $\mathcal{X}$, such as $\langle X, \succcurlyeq, \oplus\rangle$ in the above example. Think of it as the primitives

\footnotetext{
${ }^{1}$ Infamously, the mathematician Peter Freyd is reported to have once judged that the whole RTM literature was nothing but a long series of corollaries of the Hölder theorem (see, e.g., Luce, 1996, p. 82-with a rejoinder of sorts from Luce on p. 90).
} 
of a candidate measurement situation, for instance the objects of interest and the proposed operations upon them. On the other hand, take a quantitative relational structure $\mathcal{Y}$, such as $\left\langle\mathbb{R}_{>0}, \geq,+\right\rangle$ in the example. Think of it as the potential outcomes of a measurement situation, i.e., numerical results, together with a selection of the relations naturally holding between numbers. Of central interest to RTM is the set of all homomorphisms of $\mathcal{X}$ into $\mathcal{Y}$, i.e., all structure-preserving mappings between a qualitative relational structure and a quantitative relational structure of the same type. ${ }^{2}$ Call this set $\Phi$, with generic element $\phi$. The existence claim in the representation result amounts to claiming that, given the properties of $\mathcal{X}, \Phi$ is not empty. In the example, this means that given the properties of $\mathcal{X}$, there exists at least one well-defined numerical assignment $\phi$ such that $\succcurlyeq$ and $\oplus$ stand as qualitative analogues of $\geq$ and + . The uniqueness claim amounts to providing an internal characterization of $\Phi$. Indeed, when it is not empty, $\Phi$ typically contains more than one element and it needs to be clarified how any two admissible numerical assignments $\phi$ and $\psi$ relate to one another. The Hölder theorem illustrates one of the classical uniqueness categories, namely, the case of so-called ratio scales. In what follows, I will give special emphasis to the difference between the various uniqueness categories. This is in general an important topic, be it only because different uniqueness categories make different classes of numerical statements robust, or meaningful, across all the admissible ways of expressing one same set of measurement results.

Both the existence claim and the uniqueness claim come with dedicated proofs. As I will later emphasize, some aspects of the proofs can be significant and this is in effect underappreciated in the current philosophy of measurement. For future reference, I now sketch the main argument in the proof of the Hölder theorem. It is an explicit construction, that goes as follows. The properties of $\mathcal{X}$ are such that, for any $x \in X$ and $n \in \mathbb{N}$, one can find an element, denoted here by $n x$, that is $\succcurlyeq$-equivalent to $n$ copies of $x$, taken together. Besides, picking any $e \in X$ as an arbitrary reference element, one can, for any $x \in X$ and $n \in \mathbb{N}$, appropriately bound $n x$ by $m_{n} e$ and $\left(m_{n}-1\right) e$, for some $m_{n} \in \mathbb{N}$. Letting $n$ go to infinity, one gets tighter and tighter bounds. In the limit, one thus obtains a well-defined function $\phi$ satisfying the two conditions in (1). Satisfying these conditions with some other function $\psi$ amounts to picking another element $e^{\prime} \in X$ as the initial unit, and repeating the construction. Extrapolating on the characteristics of proofs like the one thus sketched, Krantz and co-authors write: "the method of constructing $\phi$ is precisely the measurement procedure" (Krantz et al., 1971, p. 15).

The question to which I now turn pertains exactly to this point: Is the RTM approach generally committed to articulating measurement procedures in its investigations of measurement? I emphasize that while this is a central question raised by the metrological critique of RTM, the question is

\footnotetext{
${ }^{2}$ For a glossary regarding homomorphisms and the like, see, e.g., Narens, 1985, Sec. 1.3.
} 
of philosophical interest beyond what an assessment of this critique requires.

\section{The Case of Unknown Measurement Procedures}

Perhaps the most striking counterexample to the view according to which RTM proceeds by investigating idealized measurement procedures is based on the so-called Alper-Narens theorem (for a full statement and a proof, see, e.g., Luce et al., 1990, Thm. 20.5, p. 120). This result is considered by many, in the RTM community, as one of the most remarkable ever produced in their field. But it is still little-known outside this community, and more work in the philosophy of science will be required to fully appreciate its significance.

Consider the class of all qualitative relational structures satisfying the following requirements. Each structure is equipped with a binary order relation. It is, qua binarily ordered structure, isomorphic to the positive real numbers (endowed with the usual order). For brevity, within this paper, call "continuum" any such structure. Notice that, given any numerical representation of any continuum, one can think of the admissible transformations of the representation as automorphisms of the qualitative structure itself. ${ }^{3}$ Now, consider the class of all continua that are homogenous and finitely unique, according to the following definitions. First, given any two points in the domain of the structure, there is at least one automorphism that maps one point into the other. This is "homogeneity". Second, there are at most finitely many points on which two automorphisms can agree without being one and the same automorphism. This is "finite uniqueness". The key implications of these two definitions are the following. The first notion rules out that any point be structurally incomparable to all others, as would be, say, an absolute maximum, minimum, or zero. For instance, in the weakly positive reals equipped with the addition operation, 0 is unlike any other number in that it is the only number such that, for any $x, x+0=0+x=x$. This illustrates a failure of homogeneity. The second notion imposes that the class of all admissible transformations be some strict subset of the class of all increasing transformations. Indeed, with forms of measurement that are unique up to any increasing transformation, such as the Mohs scale for the hardness of minerals or the Beaufort scale for the force of winds, two automorphisms could agree on infinitely many points and yet be different. This illustrates a failure of finite uniqueness. For brevity, within this paper, I will call "simple" any continuum satisfying these two requirements.

Equipped with this terminology and the previously introduced notation, one can state the Alper-Narens theorem as follows. Any simple continuum, with $\Phi$ the associated set of numerical representations, falls into exactly one

\footnotetext{
${ }^{3}$ Recall when, in the previous section, we considered replacing the initial reference element $e$ with some other element $e^{\prime}$. This is a simple illustration. The more abstract statement above is explained with great didactic care in Michell, 1990, p. $158 \mathrm{ff}$.
} 
of the following three categories, construed exclusively:

i) $\forall \phi, \psi \in \Phi, \psi=\alpha \phi$, for some $\alpha \in \mathbb{R}_{>0}$;

ii) $\forall \phi, \psi \in \Phi, \psi=\alpha \phi+\beta$, for some $\alpha \in \mathbb{R}_{>0}, \beta \in \mathbb{R}$;

iii) $\forall \phi, \psi \in \Phi, \psi=\alpha \phi+\beta$, for some $\alpha$ in a non-trivial subgroup of the multiplicative positive reals (e.g., $\left\{\bar{r}^{z} \mid \bar{r} \in \mathbb{R}_{>0}, z \in \mathbb{Z}\right\}$ ), $\beta \in \mathbb{R}$.

The first category is known as the ratio category and the second, as the interval category. They are illustrated by the customary ways in which scientists measure length and temperature, respectively. The third category is intermediate between the first two. It does not yet have a well-established name in the literature. For brevity, within this paper, I will call it the "hybrid" category. Thus, the Alper-Narens theorem is the result that any simple continuum is an interval, an hybrid, or a ratio scale.

The important point for my purposes is the sheer existence of the hybrid category. This is because, to my knowledge (or that of Alper, Narens, and any commentator of the theorem named after them), the following holds. There is, in the current state of science, no measurement procedure that would fall in this category, i.e., be such that $\alpha$ in (2) can be taken only in a non-trivial subgroup, not the full group, of the multiplicative positive reals. To my knowledge, there is not even, in RTM, a representation theorem that could give an idea of the underlying measurement procedure. In noting this, I grant for now, and till the last section of this paper, that this is what such a theorem would have to deliver. In a nutshell, one is dealing here with a measurement category with unknown measurement procedures. Indeed, thinking about what measurement procedures are possible should sometimes demand focusing away from any of the currently known measurement procedures. Now, the case of the hybrid category should not trouble the practicing scientist. But it raises the following puzzle for the philosophy and, perhaps, the metaphysics, of science. While it happens that science focuses on simple continua, why did it never stumble across the hybrid category? Is there here a pure logical possibility that would prove physically infeasible? Or has this category actually been overlooked in some past scientific contexts? Noteworthily, the existence of such a puzzling measurement category with unknown measurement procedures cannot be dismissed as an artefact of the Alper-Narens theorem focusing on continua, i.e., structures that are isomorphic to the positive reals. For instance, one would discover even more such categories if one focused, instead, on structures that are isomorphic to the positive rationals (see Cameron, 1989; Macpherson, 1996).

Admittedly, the Alper-Narens theorem is remarkable in more than one way. Narens himself, for instance, finds most remarkable the restriction, under seemingly general assumptions, to merely three measurement categories. Thus, he writes: "this theorem provides some insight into why so 
few scale types have appeared in science" (Narens, 2002, p. 55). I, for one, find most remarkable the unexpected expansion of the space of measurement categories to some categories that have not yet appeared, and might as well never appear, in scientific practice. The two interpretations differ only in emphasis. On either reading, the Alper-Narens theorem directly conflicts with the view according to which RTM contributes to the philosophy of measurement and the philosophy of science by articulating and studying measurement procedures. The result also provides a striking illustration of the kind of issues which, in the best interest of the philosophy of science, the theory of measurement can explore, when it is ready to go beyond the metrological viewpoint.

\section{The Case of Irrelevant Measurement Procedures}

I now argue that, even when RTM focuses on the case of known measurement procedures, it is sometimes in such a way that the procedures are essentially irrelevant. I illustrate this with another little-known result which would demand more philosophical attention and which I will call, in this paper, the Alper-Luce theorem (see, e.g., Luce et al., 1990, Thm. 20.7, p. 124; also Luce, 2001, Thm. 5). Initially, this result was a lemma of the AlperNarens theorem, discussed in the previous section. However, as Luce was instrumental in showing (hence my naming the result after not only Alper, who first proved the result, but also Luce), it is of self-standing interest.

Recall the definition of a continuum given in the previous section. Next, define a "translation" as an automorphism of the structure that keeps fixed either all, or none, of the points of the underlying domain. Equivalently, think of translations as admissible transformations of a given initial numerical assignment that keep fixed either all, or none, of the numerical values initially assigned. Finally, recall the definition of a simple continuum. Then, the Alper-Luce theorem can be stated as follows: a continuum is simple if and only if its translations form a so-called "homogeneous Archimedean ordered" group. Mathematically, this means two things. First, an abstract variant of the Hölder theorem can be applied to the set of translations itself. ${ }^{4}$ In essence, translations themselves can be ordered and combined with one another, like concatenable rigid rods which one would try to measure. This is the "Archimedean ordered group" part of the statement. Second, the set of translations, although generally a strict subset of the set of all automorphisms, suffices here to satisfy the condition defining homogeneity. Thus, given any two points in the domain of the structure, the set of translations is rich enough to always provide an automorphism that maps one point into the other. In particular, with respect to this set, no point proves structurally

\footnotetext{
${ }^{4}$ Specifically, it is the most abstract, i.e., group-theoretic, variant of the Hölder theorem (see, e.g., Krantz et al., 1971, Thm. 2.5, p. 53) that is relevant here.
} 
incomparable to all others. This is the "homogeneous" part.

For my purposes, the key point is that the Alper-Luce theorem applies across a very broad range of existing measurement procedures. Indeed, notice that it follows from the previous section that the Alper-Luce theorem amounts to a characterization of the ratio, hybrid, and interval categories, taken together. Focus away, for the sake of the present argument, from the unknown procedures of the hybrid category. Then, the Alper-Luce theorem can be interpreted as revealing some interesting unity across existing measurement procedures as different as that of, say, length, temperature, time, or cardinal utility, to name but a few. The primitive data required by these various kinds of measurement are obviously very different, and so are the basic operations which they traditionally involve. Accordingly, any overarching unity between them is anything but trivial. In order to reveal this unity, however, the specificities of the various measurement procedures are voluntarily abstracted away. Indeed, thinking about what unites a given class of measurement procedures should sometimes demand abstracting away from the particular features of these procedures far beyond what would qualify as an idealized account of these procedures. Now, the unity revealed by the Alper-Luce theorem might be of no interest to the practicing scientist. But it is valuable information for the philosopher of science. For instance, some authors go as far as to consider that since most attributes which we regard as truly quantitative are measured on either an interval or a ratio scale, this theorem delineates a general metaphysics of quantity. See in particular Michell, 1999, p. 208ff, where special emphasis is given to one important corollary of the theorem, namely, the one that constitutes a characterization of the ratio category, specifically. However ambitious one's philosophical interpretation of the Alper-Luce theorem, the following point will hold. The theorem uncovers some philosophically interesting unity across a wide variety of measurement procedures not in spite of, but thanks to, its consciously abstracting away from the particulars of any given procedure.

A final comment is in order. The Alper-Luce theorem vividly illustrates the significant changes that occurred in RTM after the publication of Krantz et al., 1971. One might also refer to the time elapsed between this publication and that of Luce et al., 1990, i.e., the first and the last volume of the series to which these references belong. These changes, which the pioneering work of Narens was instrumental in bringing about, have led the RTM literature to increasingly focus on the uniqueness side of representation theorems. In a nutshell, while the first main wave of RTM scholarship focused primarily on existence and only derivatively on uniqueness, the second wave reversed this emphasis. ${ }^{5}$ As Narens and Luce put it, this has been "a bit of a revolution within [the RTM revolution] itself" (Narens and Luce, 1993, p. 128). Taking

\footnotetext{
${ }^{5}$ With the benefit of hindsight, this important turn is insufficiently emphasized even in Díez, 1997a; 1997b - an otherwise remarkably comprehensive survey of the history of RTM.
} 
better account of this latter wave of RTM scholarship limits the bite of the metrological critique. This is what the present section illustrates more simply than the previous one. The illustration is simpler inasmuch as RTM is here presented as directly abstracting away from the particulars of known measurement procedures, rather than more adventurously pointing towards the location of unknown measurement procedures.

\section{The Case of Unspecified Measurement Procedures}

At this juncture, it is important to realize that the content of the two previous sections remains compatible with the following analysis. The metrological critique may not apply to the uniqueness side of the RTM literature, but - so the analysis goes - when it comes to the existence side of the literature, at least, the critique must have a bite. After all, did I not previously highlight that Krantz and co-authors seem to grant that "the method of constructing $\phi$ is precisely the measurement procedure" (Krantz et al., 1971, p. 15)? Thereby (the analysis goes on), the RTM approach, which they pioneered, seems in principle exposed to the critique according to which its existence proofs do not spell out measurement procedures in sufficient detail.

In the present section, I will argue against the substance of the preceding analysis and the philosophical perspective offered by Krantz and co-authors in the passage quoted. My argument is based on representation theorems, the existence claim of which is proved in a mathematically non-constructive fashion-non-constructive representation theorems, for short. Admittedly, there are several standards for non-constructiveness in mathematics. One standard is that a proof is non-constructive if it invokes the Axiom of Choice, or some weakening thereof (e.g., Jech, 1979). According to another standard, a proof is non-constructive if, as in the case of proofs of existence by contradiction, it establishes the existence of a certain mathematical object without showing how to construct it, or to find it, based on the pre-existing objects (e.g., Beeson, 1985). The latter standard is more stringent than the former, but it is also more intuitive in that it applies more widely. The difference between these and more refined standards will not be crucial for my purposes. Non-constructive representation theorems of all varieties seem directly excluded by Krantz and co-authors in the passage quoted above. Indeed, it is easily evidenced that they favoured thoroughly constructive proofs (e.g., Krantz et al., 1971, fn. 1 p. 42; p. 62). The case of non-constructive representation theorems was more readily explored by other contributors to RTM. Focusing on the more stringent and more applicable standard for simplicity, I now explain why non-constructive representation theorems are particularly instructive.

The following example comes from mathematical psychology and psychophysics. Consider a subject who can rank the relative intensity of some 
stimuli, but only when the difference exceeds a certain constant threshold. Standard examples are pitch height or light brightness perception. This happens to instantiate a so-called "semi-ordered" structure (Luce, 1956). A semi-order is a complete binary relation, the asymmetric part of which is transitive, but not the symmetric part, with distinctive constraints on the composition of one part with the other (specifically, on the extent of the symmetric part when composed with the asymmetric part). The specific patterns of intransitivity thus induced reflect the imperfect discrimination capacities. This contrasts with any of the structures considered hitherto, that all featured weak orders, i.e., complete and fully transitive binary relations. Now, consider a finite set $X$ and let $\succcurlyeq$ denote a binary relation over $X$, with asymmetric part $\succ$. Then it can be shown that $\succcurlyeq$ is a semi-order if and only if there exists a function $\phi: X \rightarrow \mathbb{R}_{\geq 0}$ and a constant threshold $\delta \in \mathbb{R}_{\geq 0}$ such that, for any $x, y \in X$, the following representation holds:

$$
x \succ y \Leftrightarrow \phi(x)>\phi(y)+\delta .
$$

The above representation can be compared with condition i) in representation (1), that corresponds to the particular case where $\delta=0$. This particular case can be interpreted here as indicating perfect discrimination capacities. ${ }^{6}$

The example of semi-orders is relevant to the present discussion because there are two very different proofs of the above existence result (e.g., Fishburn, 1970, Sec. 2.4, Ex. 4.18; in more detail, Fishburn, 1985, Chap. 7). One proof is, like the one previously sketched for the Hölder theorem, thoroughly constructive. It provides an algorithm for assigning numbers to the elements of $X$. The algorithm is based on the fact that a semi-order induces an associated weak order, the representation of which can be built so as to deliver the desired numerical assignment. Thus, exploiting only the semi-order properties, the algorithm leads to representation (3). By contrast, the other proof is non-constructive according to the more stringent of the previously mentioned criteria. The proof starts by recasting the existence problem as an abstract linear algebra problem. It then invokes a powerful result of this field, called the Theorem of the Alternative, to offer a proof of existence by contradiction. To wit, one branch of the alternative implies the non-existence of representation (3), which is shown to contradict the assumed semi-order properties, while the other branch of the alternative implies that the desired representation exists. Given the non-constructive nature of the argument, it can be said that although this constitutes a proof of existence, the proof leaves the underlying measurement procedure entirely unspecified. I take this lack of specification to be particularly unsatisfactory from a metrological viewpoint.

\footnotetext{
${ }^{6}$ The uniqueness properties of representation (3) is a delicate topic, especially when $X$ is finite. More details are in Suppes et al., 1989, Sec. 16.2.
} 
I do not mean to enter here into any general discussion of the respective merits of constructive and non-constructive proofs. I merely want to highlight that, as far as the specific purposes of RTM are concerned, it is often unclear whether the latter should be considered inferior to the former. Importantly, this is not merely a reflective point in the philosophy of measurement. This also applies to at least some of the empirical uses of RTM from the point of view of practicing scientists. Indeed, notice that results like the axiomatization of representation (3) have the following empirical function (other applications of such axiomatization are discussed in, e.g., Pirlot and Vincke, 1997, Chap. 2): they permit testing whether the discrimination model in representation (3) can be applied to a given subject faced with a given set of stimuli. This might be empirically unwarranted, as, for instance, when the subject's discrimination capacities are limited, but under the constraint of a threshold function that is not constant, or even deterministic, across all stimuli comparisons. This is exactly what an axiomatization of (3) permits checking. Crucially, to that end, it is immaterial whether the characterization result has been established in a constructive or a non-constructive fashion. Indeed, thinking about whether a given measurement procedure is possible need not always coincide with trying to sketch it in a stylized fashion. As a case in point, even when it leaves the potential measurement procedure entirely unspecified, an axiomatization of (3) enables one to test whether the perceptual judgments of a given subject are, in principle, measurable as they appear in a model of perception with a constant discrimination threshold. The existence results of RTM are essentially concerned with this preliminary empirical step dedicated to testing measurability. Thereby, and most strikingly when their proof is non-constructive, these results stand as useful reminders of the fact that there is more to actual measurement than the careful implementation of a measurement procedure.

Non-constructive representation theorems thus bring out a feature which they share with constructive representation theorems - when available - and that is characteristic of RTM at large. As Narens proposes to summarize it, RTM is concerned not with delivering "description[s] of empirical measurement", but with providing "qualitative, axiomatic characterizations of quantitative situations" (Narens, 2002, p. 305). This serves empirical purposes that can be related, but not reduced, to those attached to the concrete implementation of a measurement procedure.

\section{Conclusion}

According to the metrological critique of the representational theory of measurement (RTM), RTM poorly describes the measurement procedures actually followed in science, and thereby it offers the philosophy of science with limited insights on measurement. This is an invitation to refine our understanding of RTM and how it contributes to the philosophy of measurement. 
I have argued that the metrological critique is at least partly irrelevant. It is not RTM's goal to accurately describe, be it in idealized form, the measurement procedures to be found in actual scientific practice. I have illustrated this with three limit cases where, in various ways, RTM can be said to investigate measurement without providing any measurement procedure. The first two cases illustrate, from different angles, the importance of uniqueness topics in RTM. The last case illustrates the relevance of nonconstructive existence proofs in RTM. Taken together, the cases substantiate the claim according to which RTM is concerned more with measurability than with measuring, as well as they illustrate the importance of distinguishing between the former and the latter.

More generally, such cases deliver valuable, non-metrological insights for the general philosophy of science. Thus, echoing the title of Mari, 2000, they invite philosophers of measurement to go, whenever relevant, "beyond the metrological viewpoint". This is naturally not to advocate that the metrology community should change its research agenda. This is to advocate that the RTM community should keep its own, and to remind philosophers of science that they can tap either source, and still others, for complementary insights on measurement.

\section{References}

BeEson, M. (1985): Foundations of Constructive Mathematics. Heidelberg: Springer.

Cameron, P. (1989): "Groups of Order-Automorphisms of the Rationals with Prescribed Scale Type", Journal of Mathematical Psychology, 33(2), 163-171.

Decoene, S., P. Onghena, and R. Janssen (1995): "Representationalism under Attack", Journal of Mathematical Psychology, 39(2), 234-242.

Díez, J. (1997a): "A Hundred Years of Numbers. An Historical Introduction to Measurement Theory 1887-1990: Part I: the Formation Period. Two Lines of Research: Axiomatics and Real Morphisms, Scales and Invariance", Studies in History and Philosophy of Science-Part A, 28(1), 167-185.

(1997b): "A Hundred Years of Numbers. An Historical Introduction to Measurement Theory 1887-1990: Part II: Suppes and the Mature Theory. Representation and Uniqueness", Studies in History and Philosophy of Science-Part A, 28(2), 237-265.

Fishburn, P. (1970): Utility Theory for Decision Making. New York: Wiley. (1985): Interval Orders and Interval Graphs. New York: Wiley. 
Heilmann, C. (2015): "A New Interpretation of the Representational Theory of Measurement", Philosophy of Science, 82(5), 787-797.

Jech, T. (1979): The Axiom of Choice. Amsterdam: North-Holland.

Joint Committee for Guides in Metrology (2008): International Vocabulary of Metrology-Basic and General Concepts and Associated Terms. Sèvres: Joint Committee for Guides in Metrology (Third Edition).

Krantz, D., D. Luce, P. Suppes, and A. Tversky (1971): Foundations of Measurement, Volume I: Additive and Polynomial Representations. New York: Academic Press.

Luce, D. (1956): "Semiorders and a Theory of Utility Discrimination", Econometrica, 24(2), 178-191.

(1996): "The Ongoing Dialog between Empirical Science and Measurement Theory", Journal of Mathematical Psychology, 40(1), 78-98.

(2001): "Conditions Equivalent to Unit Representations of Ordered Relational Structures", Journal of Mathematical Psychology, 45(1), 81-98.

Luce, R., D. Krantz, P. Suppes, and A. Tversky (1990): Foundations of Measurement, Volume III: Representation, Axiomatization, and Invariance. San Diego: Academic Press.

Macpherson, D. (1996): "Sharply Multiply Homogeneous Permutation Groups, and Rational Scale Types", Forum Mathematicum, 8(8), 501-508.

Mari, L. (2000): "Beyond The Representational Viewpoint: A New Formalization of Measurement", Measurement, 27(2), 71-84.

Michell, J. (1990): An Introduction to the Logic of Psychological Measurement. Hillsdale: Lawrence Erlbaum.

(1999): Measurement in Psychology: A Critical History of a Methodological Concept. Cambridge: Cambridge University Press.

Mitchell, D., E. Tal, and H. Chang (2017): "The Making of Measurement: Editors' Introduction", Studies in History and Philosophy of Science-Part A, 65-66, 1-7.

Narens, L. (1985): Abstract Measurement Theory. Cambridge: M.I.T. Press.

(2002): Theories of Meaningfulness. Mahwaw: Lawrence Erlbaum Associates. 
Narens, L., And D. Luce (1993): "Further Comments on the "NonRevolution" Arising from Axiomatic Measurement Theory", Psychological Science, 4(2), 127-130.

Niederée, R. (1992): "What Do Numbers Measure?: A New Approach to Fundamental Measurement", Mathematical Social Sciences, 24(2-3), 237276.

Pirlot, M., and P. Vincke (1997): Semiorders: Properties, Representations, Applications. Heidelberg: Springer.

Stevens, S. (1968): "Measurement, Statistics, and the Schemapiric View", Science, 161(3844), 849-856.

Suppes, P., D. Krantz, R. Luce, and A. Tversky (1989): Foundations of Measurement, Volume II: Geometrical, Threshold, and Probabilistic Representations. San Diego: Academic Press.

TAL, E. (2013): "Old and New Problems in Philosophy of Measurement", Philosophy Compass, 8(12), 1159-1173.

Vessonen, E. (forthcoming): "The Complementarity of Psychometrics and the Representational Theory of Measurement", The British Journal for the Philosophy of Science. 